PHYSICAL REVIEW D 93, 089909(E) (2016)

\title{
Erratum: Isolated and binary neutron stars in dynamical Chern-Simons gravity [Phys. Rev. D 87, 084058 (2013)]
}

\section{Kent Yagi, Leo C. Stein, Nicolás Yunes, and Takahiro Tanaka}

(Received 11 April 2016; published 26 April 2016)

DOI: $10.1103 /$ PhysRevD.93.089909

In the original paper, the Chern-Simons correction to the osculating orbital elements due to the dipole-dipole interaction for the scalar field was derived in Eq. (130) by integrating out the interaction Lagrangian, which was taken to be the kinetic term for the scalar field [Eq. (122) in the original paper], using the scalar field solution. However, such a kinetic term only leads to the left-hand side of the evolution equation for the scalar field, i.e.,

$$
\square \vartheta=J,
$$

where $J$ is the effective source term of the scalar field equation [1]. To obtain the correct interaction Lagrangian, one also needs to include the source term $\left(L_{\text {source }}\right)$ that reproduces the right-hand side of the above evolution equation. The Lagrangian density for such an additional term is given by $-\vartheta J$. Therefore, Eq. (126) needs to be changed to

$$
\begin{aligned}
L_{\text {int }} & =\int\left(\mathcal{L}_{\text {kin,int }}+\mathcal{L}_{\text {source }, \text { int }}\right) d^{3} x, \\
& =\beta \mu_{1}^{j} \mu_{2}^{k} \partial_{i j}^{(1)} \partial_{i k}^{(2)} \int \frac{1}{r_{1}} \frac{1}{r_{2}} d^{3} x,
\end{aligned}
$$

where $\mathcal{L}_{\text {source,int }}$ is the interaction part of the source term Lagrangian density. Notice that, by accounting for such an additional term, the sign of the interaction Lagrangian now flips. This means that the sign in Eqs. (127), (129), and (130) needs to be reversed. The results in Sec. IX B are unaffected since we have neglected the contribution from the dipole-dipole interaction because the spin of the secondary pulsar in the double binary pulsar is much smaller than that of the primary.

We also report errors in Appendix B of our original paper. In this Appendix, we calculated dissipative corrections to the energy and angular momentum energy flux emitted from neutron star binaries based on calculations in Ref. [1]. However, some terms in the perturbed scalar and metric field equations were not properly accounted for in Ref. [1]. Taking these terms into account, we found that corrections to the gravitational energy and angular momentum flux due to metric perturbations enter at third post-Newtonian order relative to general relativity, which is of one post-Newtonian order higher than the effect due to the scalar field. Therefore, Appendixes B.1.b and B.2.b in the original paper are now irrelevant. We discuss this in more detail in Ref. [2].

On the other hand, the scalar energy flux in Appendix B.1.a is affected as follows. First, the far-zone solution for the scalar field in Eq. (B3) becomes

$$
\vartheta^{\mathrm{FZ}}=\frac{1}{r} \ddot{\mu}_{i j}\left(n^{i j}+\delta^{i j}\right)
$$

Then, the scalar energy flux in Eqs. (B5) and (B6) becomes

$$
\begin{gathered}
\delta \dot{E}^{(\vartheta)}=-\frac{8 \pi}{15} \beta\left\langle\left[\dddot{\mu}_{i j} \dddot{\mu}^{i j}+13\left(\dddot{\mu}^{i}{ }_{i}\right)^{2}\right]\right\rangle_{\omega}, \\
\delta \dot{E}^{(\vartheta)}=-\frac{5}{768} \frac{\xi_{\mathrm{CS}}}{m^{4}}\left\langle\frac { m ^ { 6 } } { r _ { 1 2 } ^ { 6 } } \left(\Delta^{2} v_{12}^{2}+27\left(\Delta \cdot v_{12}\right)^{2}\right.\right. \\
+3 \Delta^{2}\left(n_{12} \cdot v_{12}\right)^{2}-162\left(n_{12} \cdot v_{12}\right)\left(\Delta \cdot n_{12}\right)\left(\Delta \cdot v_{12}\right) \\
\left.\left.+243\left(\Delta \cdot n_{12}\right)^{2}\left(n_{12} \cdot v_{12}\right)^{2}\right)\right\rangle_{\omega},
\end{gathered}
$$


respectively. Finally, Eq. (B8) is corrected to

$$
\delta \dot{E}^{(\vartheta)}=-\frac{5}{1536} \zeta\left(\frac{m}{a}\right)^{7} \frac{29\left[\Delta_{1}^{2} f_{1}(e)+\Delta_{2}^{2} f_{2}(e)\right]+2 \Delta_{3}^{2} f_{3}(e)}{\left(1-e^{2}\right)^{11 / 2}},
$$

with Eqs. (B9) and (B10) now becoming

$$
\begin{aligned}
& f_{1}(e)=1+\frac{1129}{116} e^{2}+\frac{2271}{232} e^{4}+\frac{1287}{1856} e^{6}, \\
& f_{2}(e)=1+\frac{1075}{116} e^{2}+\frac{1731}{232} e^{4}+\frac{801}{1856} e^{6},
\end{aligned}
$$

respectively. We checked that the scalar angular momentum flux [Eq. (B24)] is not modified.

Regarding other typos in the original paper, $\bar{\mu}$ in Fig. 1 and Table 1 should be corrected to $\bar{\mu} / 4$. During the publication process, a typo was made in Eq. (B.24), which needs to be corrected as

$$
\delta \dot{L}_{i}^{(\vartheta)}=\frac{16 \pi}{15} \beta \epsilon_{i j k}\left\langle\dddot{\mu}_{j p} \ddot{\mu}_{k p}\right\rangle_{\omega} .
$$

References [93-102] were also incorrectly processed during the publication process. They should be corrected as follows: $[93] \rightarrow[96],[94] \rightarrow[97],[95] \rightarrow[98],[96] \rightarrow[99],[97] \rightarrow[100],[98] \rightarrow[101],[99] \rightarrow[102],[100] \rightarrow[93],[101] \rightarrow[94]$ and $[102] \rightarrow[95]$.

[1] K. Yagi, L. C. Stein, N. Yunes, and T. Tanaka, Phys. Rev. D 85, 064022 (2012).

[2] K. Yagi, L. C. Stein, N. Yunes, and T. Tanaka, Phys. Rev. D 93, 029902(E) (2016). 